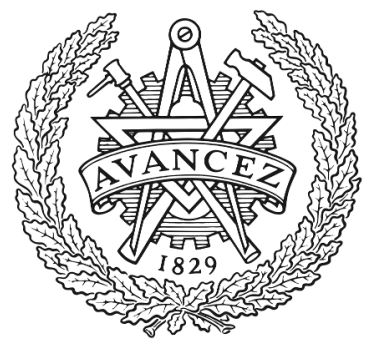

CHALMERS

UNIVERSITY OF TECHNOLOGY

\title{
Acoustic Levitation from Superposition of Spherical Harmonics Expansions of Elementary Sources: Analysis of Dependency on Wavenumber and
}

Downloaded from: https://research.chalmers.se, 2023-04-26 10:41 UTC

Citation for the original published paper (version of record):

Andersson, C., Ahrens, J. (2019). Acoustic Levitation from Superposition of Spherical Harmonics Expansions of Elementary Sources:

Analysis of Dependency on Wavenumber and Order. IEEE International Ultrasonics Symposium, IUS, 2019-October: 920-923. http://dx.doi.org/10.1109/ULTSYM.2019.8926167

N.B. When citing this work, cite the original published paper. 


\section{Acoustic Levitation from Superposition of Spherical Harmonics Expansions of Elementary Sources: Analysis of Dependency on Wavenumber and Order}

\author{
Carl Andersson \\ Division of Applied Acoustics \\ Chalmers University of Technology \\ Gothenburg, Sweden \\ Email: carl.andersson@chalmers.se
}

\author{
Jens Ahrens \\ Division of Applied Acoustics \\ Chalmers University of Technology \\ Gothenburg, Sweden \\ Email: jens.ahrens@chalmers.se
}

\begin{abstract}
Acoustic levitation of spherical objects in mid-air is a technique that is gaining traction in various human computer interaction applications such as data visualization or interactive displays. The commonly used hardware platforms are phased ultrasonic transducer arrays, used to levitate small spheres of polystyrene. Previous works have used angular spectrum decomposition of the incident sound field to derive the radiation force exerted on an arbitrary sphere. We show an alternate formulation more suited to sound fields from transducer arrays, based on direct spherical harmonics expansions of the sound fields from the individual transducer elements in the array. Finally we investigate how the truncation order of the spherical harmonics expansion influence the calculated force, for varying sphere sizes.
\end{abstract}

\section{INTRODUCTION}

Dexterous manipulation of the levitating objects in real-time requires fast calculation of the phases of the elements in the array. This is typically achieved using either simple, limited models of the radiation force along with numerical optimization, or empirical direct expressions for the element phases [1]. These methods require the levitating object to be small enough that Rayleigh scattering can be applied, i.e., $k a<1$ where $a$ is the radius of the sphere and $k=\omega / c$ is the wavenumber of the impinging waves [2]. Levitation of larger objects has previously been done using computationally expensive models to calculate the radiation force, which prevents practical realtime manipulation of objects [3].

We propose spherical harmonics based modeling of the radiation force, where the expansion coefficients are calculated directly from the known radiating elements in the array. Accurate radiation force calculation requires expansion of the pressure field from each element at, in principle, infinite orders of spherical harmonics. This is naturally not possible, and for a spherical object of finite radius there is a limiting value to which the expansions at lower orders will converge to. Using our formulation, we investigate the relation between expansion order, sphere radius, and resulting force. This is done using a

This paper is part of a project that has received funding from the European Union's Horizon 2020 research and innovation programme under grant agreement No 737087. simulation of a 16x16-element array of ultrasonic transducers, which is similar to the arrays used in previous works [4, 5, 6]. The elements are phased with a static scenario known, based on simpler models, to produce stable acoustic levitation of small beads at arbitrary positions above the array.

In this paper we describe our sound field model and subsequent radiation force model in section III followed by results from numerical simulations to investigate the relation between bead size and required spherical harmonics expansion order in section

\section{MethoD}

Our method is composed of two parts: how to efficiently obtain the spherical harmonics expansion of a sound field generated by a phased array using elementary sources, and how to calculate the radiation force on a spherical object in an arbitrary sound field using spherical harmonics expansion. Note that the last part is not dependent on the first, and can be applied to any sound field of which the spherical harmonics expansion is known.

\section{A. Sound field decomposition}

The sound field $p_{i}$ impinging on a body can be written as a sum of spherical harmonics and spherical Bessel functions, as [7]

$$
p_{i}(\vec{r})=\sum_{n=0}^{\infty} \sum_{m=-n}^{n} j_{n}(k r) S_{n, m} Y_{n m}(\theta, \varphi)
$$

where $\vec{r}$ is the position vector in the field, $S_{n, m}$ are the expansion coefficients, and the time dependency $\mathrm{e}^{-\mathrm{i} \omega t}$ is implicit. The spherical harmonics bases $Y_{n m}$ are given as

$$
Y_{n m}(\theta, \varphi)=\sqrt{\frac{2 n+1}{4 \pi} \frac{(n-m) !}{(n+m) !}} P_{n}^{m}(\cos \theta) \mathrm{e}^{\mathrm{i} m \varphi},
$$

where the associated Legendre polynomials $P_{n}^{m}$ are given as

$$
P_{n}^{m}(x)=(-1)^{m}\left(1-x^{2}\right)^{m / 2} \frac{d^{m} P_{n}(x)}{d x^{m}},
$$


and all coordinates are taken relative to the center of the levitating bead. In a similar way the scattered sound field can be written as

$$
p_{s}(\vec{r}, \omega)=\sum_{n=0}^{\infty} \sum_{m=-n}^{n} h_{n}(k r) \hat{S}_{n, m} Y_{n m}(\theta, \varphi),
$$

where $h_{n}(k r)=h_{n}^{(1)}(k a)=j_{n}(k r)+\mathrm{i} y_{n}(k r)$ are the spherical Hankel functions of the first kind. If the scattering body is a solid sphere of radius $a$ the coefficients $\hat{S}_{n, m}$ of the scattered sound field can be found as [8]

$\hat{S}_{n, m}=-\frac{j_{n}(k a) j_{n}^{\prime}\left(k_{*} a\right)-\tilde{Z} j_{n}^{\prime}(k a) j_{n}\left(k_{*} a\right)}{h_{n}(k a) j_{n}^{\prime}\left(k_{*} a\right)-\tilde{Z} h_{n}^{\prime}(k a) j_{n}\left(k_{*} a\right)} S_{n, m}=c_{n} S_{n, m}$

where $\tilde{Z}$ is the relative impedance $\left(\rho_{*} c_{*}\right) /\left(\rho_{0} c_{0}\right)$, subscript $*$ indicating the material of the sphere and subscript 0 indicate the medium, and primed symbols indicate derivatives with respect to the argument.

For an arbitrary incident sound field the expansion coefficients $S_{n, m}$ can be calculated using a spherical integration surface $\Omega$, as

$$
S_{n, m}=\frac{1}{j_{n}\left(k r_{0}\right)} \int_{\Omega} p_{i}(\vec{r}) Y_{n m}^{*}(\theta, \varphi) d \Omega
$$

where $r_{0}$ is the radius of the spherical integration surface. Note that if this equation is applied directly it is essential that the radius of the integration surface is chosen to avoid zeros of the spherical Bessel functions $j_{n}\left(k r_{0}\right)$. If the sound pressure field is created by a discrete transducer array it can be written as the superposition of the contributions $p^{j}$ from each element $j$. Since both the summation and the integral for the coefficients $S_{n, m}$ converge uniformly we can exchange the order of summation and integration as

$$
S_{n, m}=\sum_{j} \frac{1}{j_{n}\left(k r_{0}\right)} \int_{\Omega} p^{j}(\vec{r}) Y_{n m}^{*}(\theta, \varphi) d \Omega=\sum_{j} S_{n, m}^{j},
$$

i.e. the expansion coefficients of the superposed incident sound field are given by the summation of the expansion coefficients for each element in the transducer array.

Each transducer element is modeled as a point source, generating the pressure

$$
p^{j}(\vec{r})=\frac{q^{j}}{\left|\vec{r}-\vec{r}^{j}\right|} \mathrm{e}^{\mathrm{i} k\left|\vec{r}-\vec{r}^{j}\right|}
$$

where $\vec{r}^{j}$ is the location of element $j$ and $q^{j}$ is the complex source strength for the element. Modeling the transducers as point sources is a convenient choice due to the simple expressions for the expansions coefficients, see below, but there are ways to include the directivity of the transducer as well [9]. The expansion coefficients for the above expression are known to be [7]

$$
S_{n, m}^{j}=q^{j} 4 \pi \mathrm{i} k h_{n}\left(k r^{j}\right) Y_{n m}^{*}\left(\theta^{j}, \varphi^{j}\right)
$$

in the region $r<r^{j}$, i.e. closer to the scatterer than the transducer element. Note that this formulation does not require choosing a suitable radius for an integration surface, in contrast to the direct application of (6). Equations (7) and (9) are the main equations for our sound field model, and the reason why our approach is suited for transducer arrays.

\section{B. Radiation force from spherical harmonics expansions}

We use a method very similar to Sapozhnikov and Bailey to calculate the radiation force, with differences in the description of the sound field [10]. Their approach is to use a plane wave decomposition of the sound field, solving the scattering problem using spherical harmonics decompositions of each plane wave separately. As shown above, we use a solution of the scattering problem where a spherical harmonics decomposition is used directly, without any plane wave decomposition. We also use a simpler model for the scattering coefficients $c_{n}$ for our computations, but it is very easy to use other formulations of the scattering coefficients, e.g. [11, [12]. Modifying the derivations in section II D in the above mentioned paper with our description of the sound field gives the following result for the radiation force

$$
\begin{gathered}
F_{x}=\frac{1}{8 \rho_{0} c_{0}^{2} k^{2}} \Re\left\{\sum_{n=0}^{\infty} \sum_{m=-n}^{n} \Psi_{n} A_{n m}\right. \\
\left.\cdot\left(S_{n, m} S_{n+1, m+1}^{*}-S_{n,-m} S_{n+1,-m-1}^{*}\right)\right\} \\
F_{y}=\frac{1}{8 \rho_{0} c_{0}^{2} k^{2}} \Im\left\{\sum_{n=0}^{\infty} \sum_{m=-n}^{n} \Psi_{n} A_{n m}\right. \\
\left.\cdot\left(S_{n, m} S_{n+1, m+1}^{*}+S_{n,-m} S_{n+1,-m-1}^{*}\right)\right\} \\
F_{z}=\frac{1}{8 \rho_{0} c_{0}^{2} k^{2}} \Re\left\{\sum_{n=0}^{\infty} \sum_{m=-n}^{n} \Psi_{n} B_{n m}\left(S_{n, m} S_{n+1, m}^{*}\right)\right\}
\end{gathered}
$$

where

$$
\begin{aligned}
\Psi_{n} & =\mathrm{i}\left(1+2 c_{n}\right)\left(1+2 c_{n+1}^{*}\right)-\mathrm{i} \\
& =2 \mathrm{i}\left(c_{n}+c_{n+1}^{*}+2 c_{n} c_{n+1}^{*}\right) \\
A_{n m} & =\sqrt{\frac{(n+m+1)(n+m+2)}{(2 n+1)(2 n+3)}} \\
B_{n m} & =-2 \sqrt{\frac{(n+m+1)(n-m+1)}{(2 n+1)(2 n+3)}}
\end{aligned}
$$

which differers from the original result only in the definition of the expansion coefficients $S_{n, m}$ and the coefficients $\Psi_{n}$. Equations (10) and (11), supported by (7) and (9) are the main theoretical results of this paper.

\section{Comparison with previous works}

This section is a short comparison with the original equations derived by Sapozhnikov and Bailey [10]. To recover the 
original expressions, take the incident pressure as a superposition of plane waves

$$
\begin{aligned}
& p_{i}(\vec{r})= \\
& \frac{1}{4 \pi^{2}} \iint_{k_{x}^{2}+k_{y}^{2} \leq k^{2}} S\left(k_{x}, k_{y}\right) \mathrm{e}^{i\left(k_{x} x+k_{y} y+\sqrt{k^{2}-k_{x}^{2}-k_{y}^{2}} z\right)} d k_{x} d k_{y}
\end{aligned}
$$

which can be expanded as in (1) by using the addition theorem for spherical harmonics to obtain the expansion coefficients [7]

$$
S_{n, m}=\frac{\mathrm{i}^{n}}{\pi} \iint_{k_{x}^{2}+k_{y}^{2} \leq k^{2}} S\left(k_{x}, k_{y}\right) Y_{n m}^{*}\left(\theta_{k}, \varphi_{k}\right) d k_{x} d k_{y}
$$

where $\theta_{k}, \varphi_{k}$ denote the incidence angle corresponding to $k_{x}, k_{y}$, compare with (34) in [10]. Inserting this expression in (10) directly reduces to the expressions in the original work.

\section{RESULTS}

A flat transducer array with $16 \times 16$ elements in a square grid with a $1 \mathrm{~cm}$ spacing between the element centers is used for all simulations. Each transducer element is modeled as a point source, see (8), operating at $40 \mathrm{kHz}$ and with a maximum amplitude of $\left|q^{j}\right| \leq 6 \mathrm{~N} / \mathrm{m}$. The array phases are chosen to produce a sound field capable of capturing a small bead, by focusing all transducers at the desired location but including an additional phase shift of $\pi$ radians for one side of the array [5].

The radiation force is calculated using for varying radii $a$ corresponding to $k a$ between 0.05 to 50 up to order $n=50$. The target levitation location is $80 \mathrm{~mm}$ above the center of the array, and the forces are calculated $1 \mathrm{~mm}$ offset in each axis. The calculations are not performed in the center of the trap since the symmetry of the sound field causes the horizontal forces to be zero in the center of the trap.

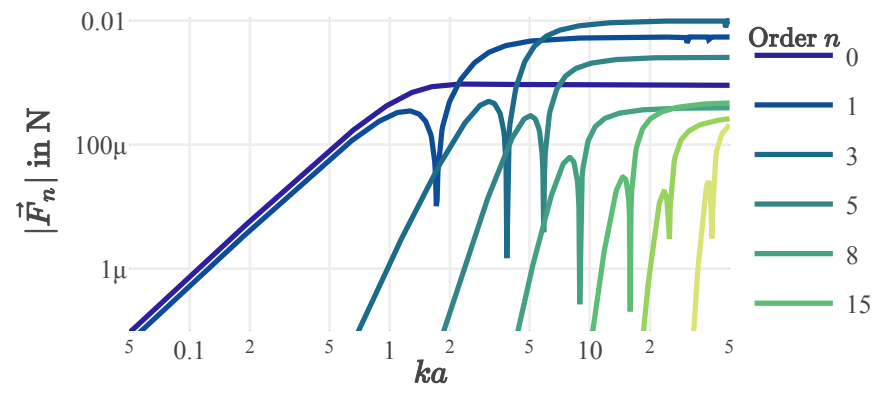

Fig. 1. Magnitude of force components from representative orders, shown over $k a$ by varying the radius of the target sphere.

From 10 we identify the partial force components $\vec{F}_{n m}$ and the force component $\vec{F}_{n}$ of a given order $n$, as

$$
\vec{F}=\sum_{n=0}^{\infty} \sum_{m=-n}^{n} \vec{F}_{n m}=\sum_{n=0}^{\infty} \vec{F}_{n}
$$

The magnitude of the force components $\left|\vec{F}_{n}\right|$ are shown for a few representative orders in Fig. 1. In a practical implementation the infinite sum needs to end after a finite number of orders $n \leq N$. The total force vector at a given truncation order $N$, i.e.

$$
\vec{F}_{N}=\sum_{n=0}^{N} \vec{F}_{n}
$$

is shown in Fig. 2 for a few orders.

From Fig. 1 it is clear that the higher order components do not play a role for smaller spheres. For $k a<n$ the force magnitude is increasing with $(k a)^{2 n+1}$, with the exception of $n=0$ which behaves like $n=1$. This behavior is expected from a small argument approximation the scattering coefficients $c_{n}$ and the coefficients $\Psi_{n}$. Similar behavior can be seen from the varying truncation orders of the force vector in Fig. 2. Comparing the lower order truncations with the higher order truncations show that a truncation of order $N$ is consistent with the higher order truncations up to $k a \approx N$, similar to results obtained in [11, pp. 427]. We can also see many resonances in the large $k a$ domain, and that for the simulated case the higher order components act to smooth out the lower order components to some extent.

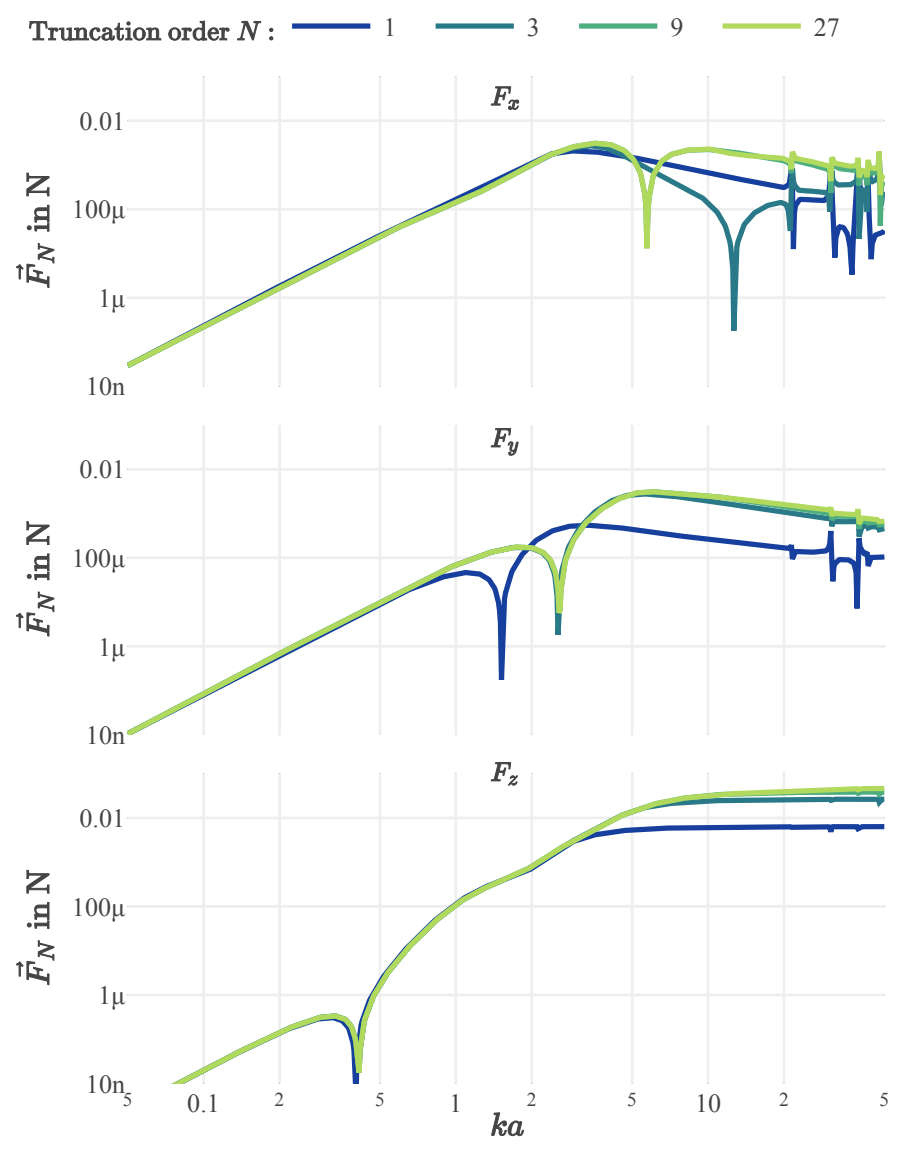

Fig. 2. Total force vector for different truncation orders $N$.

\section{Conclusions}

We have shown a fast explicit method for calculating the radiation force on spherical objects in a sound field generated 
by e.g. phased arrays. Our simulations indicate that for a given radius of the spherical object it is sufficient to truncate the spherical harmonics domain summation at approximately order $k a=N$, which confirms expectations based on previous works.

The approach is easily optimized for repeated calculation of the radiation force at a given location for different settings of the phased array by storing the constant values $S_{n, m}^{j} / q_{j}$ for each element in the array, as well as the coefficients in (11). The radiation force for a set of array element amplitudes and phases $\vec{q}$ is then calculated using simple additions and multiplications of the pre-calculated values, and no Bessel functions or spherical harmonics are evaluated. This is a structure highly suited for numerical optimizations using the array settings as the parameters of a cost function, and can be used to numerically determine array configurations to fulfill some condition, similar to what is done in [4].

\section{REFERENCES}

[1] Themis Omirou et al. "LeviPath: Modular Acoustic Levitation for 3D Path Visualisations". In: Proceedings of the 33rd Annual ACM Conference on Human Factors in Computing Systems - CHI '15 (Apr. 2015), pp. 309312.

[2] Henrik Bruus. "Acoustofluidics 7: The acoustic radiation force on small particles". In: Lab on a Chip 12.6 (2012), pp. 1014-1021.

[3] Seki Inoue et al. "Acoustical boundary hologram for macroscopic rigid-body levitation". In: Journal of the Acoustical Society of America 145.1 (Jan. 2019), pp. 328-337.

[4] Carl Andersson and Jens Ahrens. "A Method for Simultaneous Creation of an Acoustic Trap and a Quiet Zone”. In: 2018 IEEE 10th Sensor Array and Multichannel Signal Processing Workshop (SAM). IEEE, 2018, pp. 622-626.

[5] Asier Marzo et al. "Holographic acoustic elements for manipulation of levitated objects". In: Nature Communications 6.1 (2015), p. 4316.

[6] Carl Andersson and Jens Ahrens. "Minimum Trap Separation for Acoustical Levitation Using Phased Ultrasonic Transducer Arrays". In: Proceedings of the 23rd International Congress on Acoustics. Aachen, Germany, Sept. 9-13, 2019, pp. 1117-1123.

[7] Earl G Williams. Fourier Acoustics. Sound Radiation and Nearfield Acoustical Holography. Elsevier, June 1999.

[8] M F Hamilton and D T Blackstock. Nonlinear acoustics. Acoustical Society of America, 2008.

[9] Jens Ahrens. Analytic Methods of Sound Field Synthesis. Berlin, Heidelberg: Springer Berlin Heidelberg, Jan. 1, 2012. ISBN: 978-3-642-25742-1.

[10] Oleg A Sapozhnikov and Michael R Bailey. "Radiation force of an arbitrary acoustic beam on an elastic sphere in a fluid". In: Journal of the Acoustical Society of America 133.2 (Feb. 2013), pp. 661-676.
[11] N A Gumerov and R Duraiswami. Fast Multipole Methods for the Helmholtz Equation in Three Dimensions. Elsevier, Jan. 1, 2004. ISBN: 0-08-044371-0.

[12] James J Faran Jr. "Sound Scattering by Solid Cylinders and Spheres". In: Journal of the Acoustical Society of America 23.4 (June 18, 2005), pp. 405-418. DOI: 10. 1121/1.1906780. 